\title{
LEVANTAMENTO DE REQUISITOS ERGONÔMICOS PARA A CONCEPÇÃO DE ÓRTESES DE MEMBROS INFERIORES
}

\section{ERGONOMIC REQUIREMENTS SURVEY FOR THE DESIGN OF LOWER LIMB ORTHOSES}

\author{
Raquel Pizzolato Cunha de Oliveira ${ }^{1}$,Esp. \\ Flávio Anthero Dos Santos $^{2}$,Dr. \\ (1)Universidade do Estado de Santa Catarina- UDESC \\ e-mail: raquel.pizzolato@gmail.com \\ (2)Universidade do Estado de Santa Catarina-UDESC \\ e-mail: flavioanvs@hotmail.com
}

Palavras-chave: Requisitos ergonômicos, design, órteses.

\begin{abstract}
O uso de órteses é capaz de modificar a forma como a pessoa com deficiência realiza suas atividades diárias. Assim, o objetivo deste artigo foi realizar um levantamento dos requisitos ergonômicos para a concepção de órteses de membros inferiores. Mediante a revisão da literatura identificou-se que as órteses ainda apresentam significativas deficiências às demandas dos usuários. Parte disso se deve à falta de publicações abordando os requisitos que atendam inteiramente às especificações dos usuários. Em particular, há poucas indicações disponíveis para os designers sobre quais fatores devem ser atendidos durante a concepção desses. Ter os requisitos já identificados, traz como benefício agilidade no projeto, redução de tempo e custos, e dispositivos mais ergonômicos. Embora a revisão da literatura fornece uma indicação dos métodos utilizados para identificar os requisitos, não é possível determinar a partir disso quais são realmente as práticas atuais na indústria de órteses. Percebe-se que na prática ainda se priorizam os requisitos médicos e terapêuticos e poucos envolvem os usuários no processo.
\end{abstract}

\section{Keywords: Ergonomic requirements, design, orthosis.}

The use of orthoses is able to modify the way the person with disabilities performs their daily activities. Thus, the purpose of this article was to perform a survey of ergonomic requirements for the design of lower limb orthoses. Through the literature review, it was identified that the orthoses still present significant deficiencies to the users' demands. Part of this is due to the lack of publications addressing requirements that fully meet users' specifications. In particular, there are few indications available to designers about what factors should be met while designing these. Having the requirements already identified, brings as a benefit agility in the design, reduction of time and costs, and more ergonomic devices. Although the literature review provides an indication of the methods used to identify the requirements, it is not possible to determine from this what are the current practices in the orthoses industry. It is noticed that in practice, medical and therapeutic requirements are still prioritized, and few involve users in the process.

Realização:

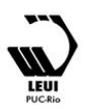

UNIVERSIDADE FEDERAL DE SANTA CATARINA 


\section{Introdução}

Faz parte da ergonomia a compreensão do comportamento e desempenho humano em sistemas de interação, afirma Wilson (2000). Deste modo, segundo o autor o papel do ergonomista divide-se em dois; o primeiro é compreender fundamentalmente as interações entre pessoas e artefatos e, especialmente, considerar as capacidades, necessidades, desejos e limitações das pessoas nessas interações. E o segundo papel compreende uma contribuição para o design de sistemas de interação, maximizando as capacidades, minimizando as limitações e tentando satisfazer as necessidades e desejos dos indivíduos.

Assim ao observar o papel da ergonomia no contexto da concepção de órteses esta disciplina auxilia em aspectos envolvendo cuidados com o corpo e sua relação com o ambiente.

As órteses, que são exemplos de Tecnologia Assistiva (TA), elas aumentam a funcionalidade e a qualidade de vida do usuário. Segundo a Academia Americana de Órteses e Próteses, o número de pessoas que usam órteses deverá aumentar de 5,6 milhões em 1995 para 7,3 milhões até 2020. Apesar do grande número de usuários, de acordo com Duarte, Mesnard e Nadeau (2016) estes dispositivos muitas vezes provam sua inadaptabilidade às demandas dos usuários.

Por serem dispositivos que ficam em contato direto com o corpo humano, a falta de adaptação ao usuário e às suas necessidades, pode resultar em problemas como a restrição de movimento e desconforto. Além disso, Duarte, Mesnard e Nadeau (2016) ressaltam o impacto em fatores sociais, afetando o usuário psicologicamente, levando ao abandono de seu uso. Por isso, a importância do fornecimento de produtos assistivos dentro dos conceitos da ergonomia, a fim $16^{\circ}$ Ergodesign - Congresso Internacional de Ergonomia e Usabilidade de Interfaces Humano Tecnológica: Produto, Informações Ambientes Construídos e Transporte

$16^{\circ}$ USIHC - Congresso Internacional de Ergonomia e Usabilidade de Interfaces Humano Computador

CINAHPA | 2017 - Congresso Internacional de Ambientes Hipermídia para Aprendizagem.

de evitar qualquer tipo de constrangimentos ao indivíduo. Diante do exposto, pretende-se neste artigo realizar o levantamento dos requisitos ergonômicos para órteses, voltado para o desenvolvimento de produtos, a fim de melhorar as interações entre o indivíduo e estes dispositivos, aumentando a eficácia destas intervenções e a qualidade de vida dos usuários.

\section{2 Órteses}

A órtese é um dispositivo aplicado externo ao corpo, com intuito de proporcionar melhora funcional ao indivíduo que sofre de algum tipo de disfunção neuromusculoesquelética. Mais especificamente, as órteses de membros inferiores, segundo as diretrizes do Ministério da Saúde (2016) são comumente indicadas para auxiliar o ortostatismo ${ }^{1}$, imobilizar segmentos articulares durante processos inflamatórios ou após intervenções cirúrgicas, prevenir, evitar e/ou corrigir deformidades, minimizar a dor e, principalmente, para permitir e facilitar uma marcha funcional e segura para a pessoa com deficiência.

Ao que diz respeito das características das órteses, estas podem ser classificadas de três maneiras; quanto a sua função, fabricação e área anatômica aplicável. Vale ressaltar que o presente estudo se dirige às órteses de membros inferiores, por motivos de delimitação da pesquisa, mais precisamente às FO (órteses para o pé) e AFO (órteses para tornozelo e pé). Contudo, as considerações apresentadas na abordagem proposta neste artigo podem ser consideradas para os demais modelos também.

\section{Requisitos ergonômicos para o desenvolvimento de órteses}

1 Posição ereta do corpo; ação de estar de
pé.
Realização:

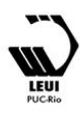

UNIVERSIDADE FEDERAL DE SANTA CATARINA 


\section{$16^{\circ}$ \\ ERGODESIGN USIHC CINAHPA}

A ergonomia tem o potencial em contribuir para a concepção de todos os tipos de sistemas de interações com pessoas, sejam sistemas de trabalho, sistemas de produtos ou serviços. Para tal, a ergonomia baseia-se, em primeiro lugar, nas capacidades humanas e mesmo nos seus limites, para conceber intervenções adaptadas às características dos indivíduos. Logo, o estudo de fatores ergonômicos aliado ao design busca criar produtos e elementos adaptados às características apresentadas pelos usuários finais. Ou seja, o processo de design deve considerar os traços físicos, mentais e psicológicos desses indivíduos.

Determinar quais necessidades devem ser satisfeitas pelo produto final caracteriza uma das primeiras atividades a serem executadas no desenvolvimento de produtos (TANURE, 2015). Então, após a identificação das necessidades é possível definir os requisitos. Young (2004 apud TANURE, 2015) descreve requisito como:

"Um atributo necessário em um sistema, uma característica ou fator de qualidade com valor e utilidade para um usuário ou usuário" (YOUNG, 2004 apud TANURE, 2015, p.57).

Em geral, um requisito é uma especificação da necessidade do usuário e contém informações relativas à solução para o problema. Sendo assim, a identificação dos requisitos é importante porque estes fornecem a base para todo o processo de desenvolvimento que se segue. Caso as necessidades do usuário e os problemas não forem totalmente compreendidos, significa que a especificação dos requisitos do sistema será incorreta, explica Gausepohl (2008) e como resultado final, o designer cria um produto que não responde às necessidades dos usuários. Por isso em cada iteração com o usuário, o produto deve ser avaliado com relação ao cumprimento dos requisitos de projeto e o resultado desta avaliação serve de base para a próxima iteração (TENURE, 2015).

Assim, o desenvolvimento ergonômico de produtos é caracterizado por um processo iterativo, um método cíclico de projeto e avaliação. Isso $16^{\circ}$ Ergodesign - Congresso Internacional de Ergonomia e Usabilidade de Interfaces Humano Tecnológica: Produto, Informações Ambientes Construídos e Transporte

$16^{\circ}$ USIHC - Congresso Internacional de Ergonomia e Usabilidade de Interfaces Humano Computador

CINAHPA | 2017 - Congresso Internacional de Ambientes Hipermídia para Aprendizagem.

permite a otimização do sistema no sentido de um loop de controle, o feedback loop, com relação ao valor de saída e a avaliação da sua eficiência e eficácia no uso real do produto. Este processo permite organizar e oferecer uma sequência de ações que possibilitam que o design seja desenvolvido de forma mais assertiva.

Conforme Gausepohl, (2008) os requisitos são geralmente divididos em duas categorias; requisitos funcionais e não funcionais. Deste modo, o autor apresenta esta divisão relacionandoa com os requisitos gerais para órteses:

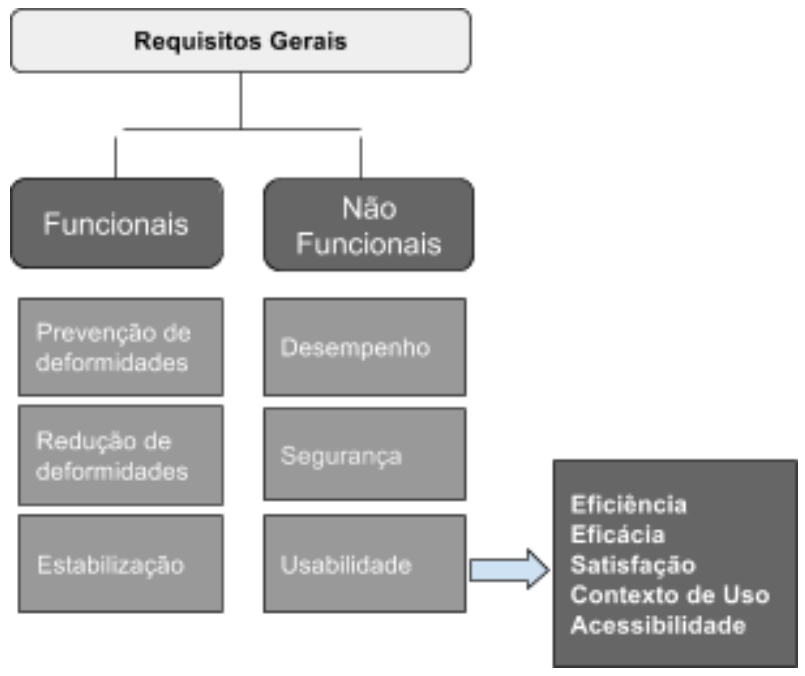

Diagrama 1: Requisitos gerais para órteses. Fonte: Gausepohl, 2008. Adaptado pelos autores, 2017.

Os requisitos funcionais de uma órtese são aqueles necessários para alcançar os objetivos clínicos previamente definidos. Estes especificam as operações do sistema. Por outro lado, os requisitos não funcionais especificam atributos de qualidade, metas e restrições do sistema (GAUSEPOHL, 2008).

Contudo, na prática atual o desenvolvimento das órteses são geralmente realizados por profissionais da área da saúde em resposta a uma necessidade médica e física. Consequentemente vários requisitos ergonômicos são deixados de lado. Para Soares (2010) o motivo disso é a priorização dos requisitos médicos e terapêuticos, ou seja, a
Realização:

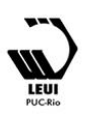

UNIVERSIDADE FEDERAL DE SANTA CATARINA
INSTITUTO FEDERAL

Santa Catarina

Câmpus Palhoça Bilíngue 


\section{$16^{\circ}$ \\ ERGODESIGN USIHC CINAHPA}

$16^{\circ}$ Ergodesign - Congresso Internacional de Ergonomia e Usabilidade de Interfaces Humano Tecnológica: Produto, Informações Ambientes Construídos e Transporte

$16^{\circ}$ USIHC - Congresso Internacional de Ergonomia e Usabilidade de Interfaces Humano Computador

CINAHPA | 2017 - Congresso Internacional de Ambientes Hipermídia para Aprendizagem. priorização do que seriam os requisitos funcionais, em vista dos requisitos não funcionais, tais como a usabilidade, satisfação, aspirações pessoais, entre outros. A abordagem para satisfazer os requisitos do usuário no desenvolvimento de órteses requer atenção para muitos fatores diferentes, demandando várias competências e a interação entre disciplinas distintas que têm de convergir para um objetivo comum. Na tentativa de melhorar os produtos de tecnologia assistiva os autores Batavia e Hammer (1990) realizaram um estudo utilizando o método Delphi. Este método de pesquisa é projetado para facilitar a construção de consenso entre pequenos grupos de especialistas, e para identificar os requisitos priorizados pelos usuários com algum tipo de deficiência.

No total, Batavia e Hammer (1990) identificaram 17 fatores gerais para as tecnologias assistivas, apresentados a seguir, juntamente com a pergunta que deve ser feita de modo a identificar se o dispositivo adota ou não o requisito;

1. Viabilidade. A compra, manutenção e/ou reparação do dispositivo causa dificuldades financeiras ou dificuldades ao usuário?

2. Compatibilidade. Como o dispositivo irá interagir com outros dispositivos?

3. Reparabilidade (do usuário). O usuário consegue reparar o dispositivo?

4. Confiabilidade. O dispositivo opera com níveis de precisão repetíveis e/ou previsíveis em todas as condições de uso razoável?

5. Durabilidade. O dispositivo continuará a ser operável por um longo período de tempo?

6. Facilidade de montagem. O usuário pode facilmente montar o dispositivo ao recebê-lo?

7. Facilidade de manutenção. $\mathrm{O}$ usuário pode facilmente custear o dispositivo para mantê-lo operacional e seguro? É fácil realizar todos os procedimentos necessários de manutenção, limpeza e controle de infecção?

8. Eficácia. $\mathrm{O}$ funcionamento do dispositivo melhora a situação de vida do indivíduo, tal como esta é percebida pelo usuário incluindo se aumenta a capacidade funcional e /ou a independência deste?
9. Flexibilidade. O dispositivo é fornecido com opções disponíveis a partir das quais o usuário pode escolher.

10. Aprendizagem. Ao receber inicialmente o dispositivo, o usuário pode facilmente aprender a usá-lo e pode começar a usá-lo dentro de um período de tempo razoável, uma vez montado, incluindo se é necessário treinamento especializado?

11. Operabilidade. O dispositivo é fácil de operar e responde adequadamente aos comandos operacionais do usuário?

12. Aceitação pessoal. É psicologicamente confortável usar o dispositivo em público (ou em particular)? O dispositivo é esteticamente atraente?

13. Conforto físico. O dispositivo provoca dor física ou desconforto para o usuário?

14. Segurança física. O dispositivo é suscetível de causar danos físicos, incluindo lesões corporais ou infecção, ao usuário?

15. Portabilidade. O dispositivo pode ser facilmente transportado para ser operado em locais diferentes? O tamanho e peso do dispositivo permitem a deslocação física?

16. Segurança (contra furtos). $O$ dispositivo pode ser mantido facilmente dentro do controle físico do usuário para reduzir a probabilidade de roubo ou vandalismo?

17. Reparabilidade (do fornecedor). Até que ponto um fornecedor local ou uma oficina de reparo pode reparar o dispositivo dentro de um período razoável de tempo? As peças de reposição estão prontamente disponíveis caso seja necessário realizar reparos?

Estes fatores identificados por Batavia e Hammer (1990) podem constituir em um guia de requisitos para o projeto de órteses. Fornecendo informações valiosas sobre critérios a serem utilizados na concepção e aquisição de dispositivos de tecnologia assistiva.

É essencial que os profissionais que estiverem envolvidos no processo de desenvolvimento e prescrição destes dispositivos reconheçam os fatores que são importantes para os usuários, pois,
Realização:
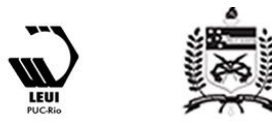

UNIVERSIDADE FEDERAL DE SANTA CATARINA
INSTITUTO FEDERAL

Santa Catarina

Câmpus Palhoça Bilíngue 


\section{$16^{\circ}$ \\ ERGODESIGN USIHC CINAHPA}

$16^{\circ}$ Ergodesign - Congresso Internacional de Ergonomia e Usabilidade de Interfaces Humano Tecnológica: Produto, Informações Ambientes Construídos e Transporte

$16^{\circ}$ USIHC - Congresso Internacional de Ergonomia e Usabilidade de Interfaces Humano Computador

CINAHPA | 2017 - Congresso Internacional de Ambientes Hipermídia para Aprendizagem. a falta de consideração desses requisitos resultará na contínua insatisfação, frustração e provavelmente no abandono do dispositivo pelo usuário. Em vista disso, Zhao e Phillips (1993) realizaram uma pesquisa para entender quais fatores estavam relacionados ao abandono das tecnologias assistivas. Eles identificaram quatro fatores mais recorrentes; falta de consideração pela opinião do usuário; procura de aparelho fácil; desempenho ruim do dispositivo; mudança na necessidade ou prioridade do usuário. Três dos quatro motivos de abandono citados estão relacionados a problemas envolvendo a má concepção do projeto, ou seja, a raiz do problema é que muitos destes dispositivos foram concebidos em torno da incapacidade do usuário,

desconsiderando como esses usuários vivem suas vidas diárias, seus desejos e expectativas, ou seja sem ter um enfoque pleno sob os requisitos ergonômicos. Estudos como o de Zhao e Phillips (1993) proporcionam uma ampla visão de como a consideração destes fatores são importantes no uso bem-sucedido ou não dos recursos de tecnologia assistiva. Tais dispositivos se tornam inutilizáveis quando não há a melhora da qualidade de vida do utilizador e são descartados.

\section{Considerações Finais}

A revisão da literatura sobre a concepção de órteses, revelou que existem poucos trabalhos publicados sobre os aspectos ergonômicos do desenvolvimento destes dispositivos. Em particular, há poucas indicações disponíveis para os designers sobre quais fatores devem ser atendidos durante a concepção e desenvolvimento, ou recomendações de boas práticas em termos de métodos e abordagens necessárias para capturar toda a gama de requisitos técnicos e do usuário. Durante a realização deste artigo foi encontrada somente uma única publicação (BATAVIA e HAMMER, 1990) que faz a enumeração de requisitos necessários e que podem ser aplicados para a concepção de órteses de membros inferiores. As demais publicações enfocam na descrição dos métodos para identificação dos requisitos, sem chegar a de fato na descrição dos resultados obtidos, ou seja, nos requisitos identificados. A relevância de fazer o levantamento destes requisitos se dá pelo fato destes mostrarem quais necessidades devem ser satisfeitas pelo produto final. Ter um guia com os principais requisitos já identificados, traz como benefício agilidade no projeto, redução de tempo e custos, além de dispositivos que atendam melhor às necessidades dos usuários. Embora a revisão da literatura fornece uma indicação dos métodos que foram usados para identificar os requisitos, não é possível determinar a partir disso quais são realmente as práticas atuais na indústria de órteses. Percebe-se que na prática ainda se priorizam os requisitos médicos e terapêuticos e poucos envolvem os usuários no processo. Além disso, muitos dos métodos discutidos nestes estudos requerem uma intensiva mão de obra para captar as necessidades dos usuários e muito recurso de tempo, tornandoos pouco aplicáveis na prática real. Outro ponto observado durante a revisão, foi a tendência de estudos que abordam a relevância do envolvimento do usuário em cada etapa do processo de desenvolvimento das órteses a fim de atingir uma melhor performance. Contudo, as pesquisas revelam que ainda existe um longo caminho para que o desenvolvimento de órteses de membros inferiores adquira o desempenho ideal. Um dos supostos motivos para a presente deficiência é a falta de interdisciplinaridade no desenvolvimento destas pesquisas, fazendo assim que muitos fatores importantes sejam deixados de lado, pois não são de responsabilidade daquela disciplina em específico. Portanto a função do designer é um elemento fundamental na concepção e desenvolvimento de órteses, de modo que ele é capaz de integrar a adequação das funções do produto com as diferentes necessidades dos usuários. Para futuras pesquisas se sugere relacionar cada um dos 17 fatores descritos por Batavia e Hammer (1990) à concepção de órteses de membros inferiores, a fim de produzir um guia de requisitos específicos para o desenvolvimento de órteses de membros inferiores, de modo que agilize e melhore o processo de desenvolvimento destas.
Realização:

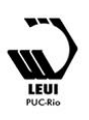




\section{$16^{\circ}$ \\ ERGODESIGN USIHC CINAHPA}

$16^{\circ}$ Ergodesign - Congresso Internacional de Ergonomia e Usabilidade de Interfaces Humano Tecnológica: Produto, Informações Ambientes Construídos e Transporte

$16^{\circ}$ USIHC - Congresso Internacional de Ergonomia e Usabilidade de Interfaces Humano Computador

CINAHPA | 2017 - Congresso Internacional de Ambientes Hipermídia para Aprendizagem.

\section{Referências}

BATAVIA, A.I., HAMMER, G.S. Toward the development of consumer-based criteria for the evaluation of assistive devices. J. Rehabil. Res. Dev. 27, 425-436,1990.

BRASIL. Ministério da Saúde. Diretrizes para prescrição, concessão, adaptação e manutenção de órteses, próteses e meios auxiliares de locomoção. OPM do Sistema Único de Saúde.Consulta Pública publicada 01 de abril de 2016

DUARTE, R.; MESNARD, M.; NADEAU, J.P. An innovative design approach to develop external articular medical devices. International Journal on Interactive Design and Manufacturing (IJIDeM), p. 1-9, 2016.

GAUSEPOHL, K.A. Investigation of Storytelling as a Requirements Elicitation Method for Medical Devices. Master of Science in Industrial and Systems Engineering. Faculty of the Virginia
Polytechnic Institute and State University .Blacksburg, USA.2008

SOARES, M.M. Translating user needs into product design for the disabled: an ergonomic approach. Theoretical Issues in Ergonomics Science, v.13, n.1, p.92-120, 2012.

TANURE, R.L.Z. Análise multidimensional de requisitos funcionais e emocionais para avaliação de produtos na perspectiva do usuário. Tese (Doutorado em Engenharia) - Pós Graduação em Engenharia de Produção da Universidade Federal do Rio Grande do Sul. Porto Alegre. 2015

WILSON, J.R; Fundamentals of ergonomics in theory and practice. Fundamental Reviews in Applied Ergonomics. v31, n.6, p. 557-567, 2000.

ZHAO, H.; PHILLIPS, B. Predictors of assistive technology abandonment. Assist. Technol., v. 5, n. 1, p. 35-45, 1993.
Realização:

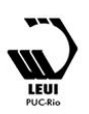

